\title{
Correction to: A 10 Step Framework to Implement Integrated Care for Older Persons
}

Patrick John Harnett ${ }^{1} \cdot$ Siobhan Kennelly ${ }^{2} \cdot$ Peter Williams $^{3}$

Published online: 14 October 2019

(C) Springer Science+Business Media, LLC, part of Springer Nature 2019

\section{Correction to: Ageing International https://doi.org/10.1007/s12126-019-09349-7}

The original version of this article was revised. The table below shows the incorrect and correct columns.

\section{Patrick John Harnett}

pjharnett.sdu@hse.ie

Siobhan Kennelly

siobhan.kennelly1@hse.ie

Peter Williams

peter.williams@ul.ie

1 Social Care Division/Clinical Strategy and Programme Division, Health Service Executive, Dublin, Ireland

2 Clinical Strategy and Programme Division/Social Care Division, Health Service Executive, Dublin, Ireland

3 Department of Research Engineering, University of Limerick, Limerick, Ireland 
Page No.

Incorrect

Correct

Section

Paragraph No.

Line No.

Page 1

Abstract

Paragraph 1

Line 18

Page 2

Background

Paragraph 1

Line 16

Page 2

Background

Paragraph 1

Line 27

Page 2

Introduction

Paragraph 2

Line 4

Page 4

Methods

Paragraph 2

Line 11

Page 5

The Challenge of Implementation

Page 5

The Challenge of Implementation

Page 6

The Challenge of Implementation

Page 6

The Challenge of Implementation

Page 7

The Challenge of Implementation

Page 8

Results

Page 9

Results

Paragraph 2

Line 7

Page 9

Results

Paragraph 2

Line 8

Page 9

Results

Paragraph 4

Line 7 the change methodology fails

is centrally defined

In doing so

2500 bed hospitals

No figure placed after Methods paragraph

Fig. 3

Fig. 4

Fig. 5

Fig. 6

Fig. 7

Fig. 8

Fig. 6 citation

no citation after the very last word

no citation after the very last word with most change methodologies fail

is typically centrally defined
500 bed hospitals

10 Step Framework (Fig.3) should be placed after Methods paragraph

relabel as Fig. 6 and move to section indicated

relabel as Fig. 7 and move to section indicated

relabel as Fig. 9 and move to section indicated

relabel as Fig. 10 and insert to section indicated

relabel as Fig. 3 move to section indicated

relabel as Fig. 4 move to section indicated

should be removed

insert Fig. 5 citation

insert Table 1 citation 


Page No. $\quad$ Incorrect
Section
Paragraph No.
Line No.

\section{Page 9}

Results

Paragraph 5

Line 3

Page 9

Results

Paragraph 7

after line 4

Page 10

Results

Paragraph 7

after line 12

Page 11

Results

Paragraph 8

after line 4

\section{Page 11}

Discussion

Paragraph 1

after line 21

\section{Page 12}

Results

Paragraph 2

Line 11

Page 12

Results

Paragraph 2

Line 14

Page 12

Results

Paragraph 3

Line 9 no citation after the very insert Fig. 6 citation
last word

Fig. 9

relabel as Fig. 5

no citation after the very last insert Fig. 7 citation
word

no citation after the very last insert Fig. 8 citation word

Fig. 11

relabel as Fig. 8

Fig. 5. citation should be citation Fig. 9

no figure below the very last place Fig. 9 word

no citation after the very last insert Fig. 10 citation word

Publisher's Note Springer Nature remains neutral with regard to jurisdictional claims in published maps and institutional affiliations. 